\title{
Capacity Region of the Broadcast Channel with Two Deterministic Channel State Components
}

\author{
Hyeji Kim and Abbas El Gamal \\ Department of Electrical Engineering \\ Stanford University \\ Email: hyejikim@stanford.edu, abbas@ee.stanford.edu
}

\begin{abstract}
This paper establishes the capacity region of a class of broadcast channels with random state in which each channel component is selected from two possible functions and each receiver knows its state sequence. This channel model does not fit into any class of broadcast channels for which the capacity region was previously known and is useful in studying wireless communication channels when the fading state is known only at the receivers. The capacity region is shown to coincide with the UV outer bound and is achieved via Marton coding.
\end{abstract}

\section{INTRODUCTION}

The 2-receiver broadcast channel with two deterministic channel states (or BC-TDCS in short) is a discrete memoryless broadcast channel with random state $(\mathcal{X} \times$ $\left.\mathcal{S}, p(s) p\left(y_{1}, y_{2} \mid x, s\right), \mathcal{Y}_{1} \times \mathcal{Y}_{2}\right)$, where $S=\left(S_{1}, S_{2}\right) \in\{1,2\}^{2}$, $p_{S_{1}}(1)=p_{1}, p_{S_{1}}(2)=1-p_{1}=\bar{p}_{1}$ and $p_{S_{2}}(1)=p_{2}, p_{S_{2}}(2)=$ $\bar{p}_{2}$, and the outputs

$$
\begin{aligned}
& Y_{1}= \begin{cases}f_{1}(X) & \text { if } S_{1}=1, \\
f_{2}(X) & \text { if } S_{1}=2,\end{cases} \\
& Y_{2}= \begin{cases}f_{1}(X) & \text { if } S_{2}=1, \\
f_{2}(X) & \text { if } S_{2}=2\end{cases}
\end{aligned}
$$

for some deterministic functions $f_{1}$ and $f_{2}$ of the input $X$. As an example of a BC-TDCS, consider the following.

Example 1 (Blackwell channel with state [1]). The functions $f_{1}$ and $f_{2}$ for this example are depicted in Figure 1

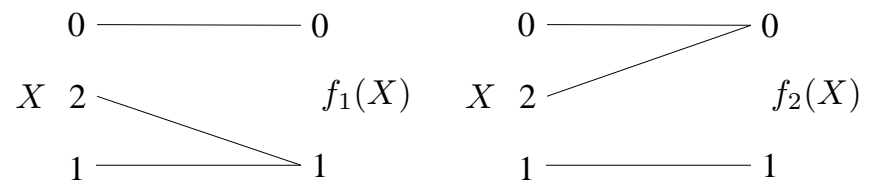

Fig. 1. The deterministic components of the Blackwell channel with state.

In this paper, we consider the setup in which the sender wishes to transmit an independent message $M_{j} \in\left[1: 2^{n R_{j}}\right]$ to receiver $j \in\{1,2\}$ and receiver $j$ knows the state sequence $S_{j}^{n}$ but the sender does not. We define achievable rate pairs $\left(R_{1}, R_{2}\right)$ in the standard way [2] and the capacity region $\mathrm{C}$ as the closure of the set of all achievable rate pairs.

It is easy to see that the capacity region of this channel is the same as that of the broadcast channel with input $X$ and outputs $\left(Y_{1}, S_{1}\right)$ and $\left(Y_{2}, S_{2}\right)$. This equivalent broadcast

This work was partially supported by Air Force grant FA9550-10-1-0124. channel, however, does not belong to any class of channels with known capacity region (see [3] for classes of broadcast channels with known capacity). Also, very little is known about the capacity region of the broadcast channel with random state known only at the receivers. Previous work on this setting has focused mainly on the Gaussian fading BC with superposition coding [4], time division with power control [5], and a superposition of binary inputs motivated by a capacity achieving strategy for a layered erasure broadcast channel [6]. Even when the fading $\mathrm{BC}$ is degraded and superposition coding is optimal, Gaussian input distribution is not in general optimal and capacity remains unknown [7].

There has been more work on the broadcast channel with causal and noncausal state information known at the transmitter. In [8], the capacity region of the deterministic BC when the state is known noncausally at the transmitter is established. In [9], this result is extended to semideterministic BC, and it is shown that the capacity region does not enlarge when the state is also known at the receivers.

There has also been work on the setting in which the state is known at the receivers and only strictly causally at the transmitter. In [10], [11], the capacity region of the binary erasure broadcast channel with state under this setting is established. In [12] it is shown via two examples that strictly causal state information at the transmitter can enlarge the capacity region of the broadcast channel with state. In [1], it is shown that the scheme in [12] is a special case of a straightforward adaptation of the feedback scheme in [13]. The Blackwell broadcast channel with state in Example 1 is also introduced and an achievable rate region is established when $p_{1}=p_{2}=0.5$ (and the state in known at the receivers and strictly casually at the transmitter).

In this paper we establish the capacity region of the BC-TDCS when the state is known only at the receivers. Achievability is established using Marton coding [14]. The key observation is that the auxiliary random variables in the Marton region characterization, $U_{1}$ and $U_{2}$, are always set to $f_{1}, f_{2}, X$, or $\emptyset$. In particular if the channel from $X$ to $Y_{1}$ is more likely to be $f_{1}$ than the channel from $X$ to $Y_{2}$, then $\left(U_{1}, U_{2}\right)$ are set to $(X, \emptyset),(\emptyset, X)$, or $\left(f_{1}, f_{2}\right)$. The converse is established by showing that the Marton inner bound with these extreme choices of auxiliary random variables coincides with the UV outer bound [15].

Our result is significant for several reasons: 
- It establishes the capacity region of a new class of broadcast channels-our setting does not belong to any class of broadcast channels with previously known capacity region.

- It establishes the capacity region of a nontrivial class of broadcast channels with state known at the receivers-a setting with very few known results.

- It provides yet another class of broadcast channels for which Marton coding is optimal.

- Our channel model can be used to approximate certain fading broadcast channels in high SNR (see Example 2 in Section (1).

\section{Capacity Region of the BC-TDCS}

Without loss of generality, assume $p_{1} \geq p_{2}$. We now state the main result of this paper.

Theorem 1. The capacity region of the BC-TDCS $(\mathcal{X} \times$ $\left.\mathcal{S}, p(s) p\left(y_{1}, y_{2} \mid x, s\right), \mathcal{Y}_{1} \times \mathcal{Y}_{2}\right)$ with the state known only at the receivers is the convex hull of the set of all rate pairs $\left(R_{1}, R_{2}\right)$ such that

$$
\begin{aligned}
R_{1} & \leq I\left(U_{1} ; Y_{1} \mid S\right) \\
R_{2} & \leq I\left(U_{2} ; Y_{2} \mid S\right), \\
R_{1}+R_{2} & \leq I\left(U_{1} ; Y_{1} \mid S\right)+I\left(U_{2} ; Y_{2} \mid S\right)-I\left(U_{1} ; U_{2}\right)
\end{aligned}
$$

for some $p(x)$ and either $\left(U_{1}, U_{2}\right)=\left(f_{1}, f_{2}\right),\left(U_{1}, U_{2}\right)=$ $(X, \emptyset)$, or $\left(U_{1}, U_{2}\right)=(\emptyset, X)$.

Achievability follows immediately since (1) is contained in Marton's rate region. The converse is proved in Section III

Now consider the following more explicit characterization of the capacity region which we will use in the examples and the converse.

Proposition 1. The capacity region of the BC-TDCS with the state known only at the receivers is the convex hull of the union of four rate regions:

$$
\begin{aligned}
\mathcal{R}_{1}=\left\{\left(R_{1}, R_{2}\right):\right. & \left.R_{1} \leq C_{1}, R_{2}=0\right\} \\
\mathcal{R}_{2}=\left\{\left(R_{1}, R_{2}\right):\right. & \left.R_{1}=0, R_{2} \leq C_{2}\right\} \\
\mathcal{R}_{3}=\left\{\left(R_{1}, R_{2}\right):\right. & R_{1} \leq p_{1} H\left(f_{1}\right)+\bar{p}_{1} I\left(f_{1} ; f_{2}\right), \\
& R_{2} \leq \bar{p}_{2} H\left(f_{2} \mid f_{1}\right) \\
& \text { for some } \left.p(x) \in \mathcal{P}_{1}\right\}, \\
\mathcal{R}_{4}=\left\{\left(R_{1}, R_{2}\right):\right. & R_{1} \leq p_{1} H\left(f_{1} \mid f_{2}\right), \\
& R_{2} \leq p_{2} I\left(f_{1} ; f_{2}\right)+\bar{p}_{2} H\left(f_{2}\right) \\
& \text { for some } \left.p(x) \in \mathcal{P}_{2}\right\},
\end{aligned}
$$

where $C_{j}=\max _{p(x)} I\left(X ; Y_{j} \mid S\right)$ for $j=1,2$, and

$$
\begin{aligned}
& \mathcal{P}_{1}=\left\{\underset{p(x)}{\arg \max } p_{1} H\left(f_{1}\right)+\bar{p}_{1} I\left(f_{1} ; f_{2}\right)+\lambda \bar{p}_{2} H\left(f_{2} \mid f_{1}\right):\right. \\
&\text { for some } \left.\bar{p}_{1} / \bar{p}_{2} \leq \lambda \leq 1\right\}, \\
& \mathcal{P}_{2}=\left\{\underset{p(x)}{\arg \max } p_{1} H\left(f_{1} \mid f_{2}\right)+\lambda p_{2} I\left(f_{1} ; f_{2}\right)+\lambda \bar{p}_{2} H\left(f_{2}\right):\right.
\end{aligned}
$$

for some $\left.1 \leq \lambda \leq p_{1} / p_{2}\right\}$.

Proof: Let $\mathrm{C}$ and $\mathrm{C}_{0}$ denote the region defined in (1) and in (2), respectively. All we need to show is that $\mathcal{C}_{0}=\mathcal{C}$. First note that we can express $\mathcal{C}$ as the convex hull of the union of the four regions:

$\mathcal{R}_{1}^{\prime}=\left\{\left(R_{1}, R_{2}\right): R_{1} \leq I\left(X ; Y_{1} \mid S\right), R_{2}=0\right.$ for some $\left.p(x)\right\}$,

$\mathcal{R}_{2}^{\prime}=\left\{\left(R_{1}, R_{2}\right): R_{1}=0, R_{2} \leq I\left(X ; Y_{2} \mid S\right)\right.$ for some $\left.p(x)\right\}$,

$\mathcal{R}_{3}^{\prime}=\left\{\left(R_{1}, R_{2}\right): R_{1} \leq I\left(f_{1} ; Y_{1} \mid S\right)\right.$,

$R_{2} \leq I\left(f_{2} ; Y_{2} \mid S\right)-I\left(f_{1} ; f_{2}\right)$

for some $p(x)\}$,

$\mathcal{R}_{4}^{\prime}=\left\{\left(R_{1}, R_{2}\right): R_{1} \leq I\left(f_{1} ; Y_{1} \mid S\right)-I\left(f_{1} ; f_{2}\right)\right.$,

$R_{2} \leq I\left(f_{2} ; Y_{2} \mid S\right)$

for some $p(x)\}$.

Clearly $\mathcal{R}_{1}=\mathcal{R}_{1}^{\prime}, \mathcal{R}_{2}=\mathcal{R}_{2}^{\prime}, \mathcal{R}_{3} \subseteq \mathcal{R}_{3}^{\prime}$, and $\mathcal{R}_{4} \subseteq \mathcal{R}_{4}^{\prime}$. Thus, $\mathcal{C}_{0} \subseteq \mathcal{C}$. We now show that every supporting hyperplane of $\mathcal{C}$ intersects $\mathcal{C}_{0}$, i.e., for every $\lambda \geq 0$, there exists a rate pair $\left(R_{1}, R_{2}\right) \in \mathcal{C}_{0}$ such that $R_{1}+\lambda R_{2}=\max _{\left(R_{1}, R_{2}\right) \in \mathcal{C}} R_{1}+\lambda R_{2}$.

Lemma 1. Every supporting hyperplane of $\mathcal{C}$ intersects $\mathcal{C}_{0}$, i.e., for all $\lambda \geq 0$,

$$
\max _{\left(R_{1}, R_{2}\right) \in \mathrm{e}} R_{1}+\lambda R_{2}=\max _{\left(R_{1}, R_{2}\right) \in \mathcal{C}_{0}} R_{1}+\lambda R_{2} .
$$

The proof of this lemma is in Appendix A

To complete the proof we use the following.

Lemma 2. [16] Let $\mathcal{R} \in \mathbb{R}^{d}$ be convex and $\mathcal{R}_{1} \subseteq \mathcal{R}_{2}$ be two bounded convex subsets of $\mathcal{R}$, closed relative to $\mathcal{R}$. If every supporting hyperplane of $\mathcal{R}_{2}$ intersects $\mathcal{R}_{1}$, then $\mathcal{R}_{1}=\mathcal{R}_{2}$.

Example 1 (continued) The capacity region of the Blackwell channel with state known only to the receivers is the convex hull of the union of:

$$
\begin{aligned}
\mathcal{R}_{3}^{\prime}=\left\{\left(R_{1}, R_{2}\right):\right. & R_{1} \leq H\left(\alpha_{0}\right)-\bar{p}_{1} \bar{\alpha}_{1} H\left(\alpha_{0} / \bar{\alpha}_{1}\right) \\
& R_{2} \leq \bar{p}_{2} \bar{\alpha}_{0} H\left(\alpha_{1} / \bar{\alpha}_{0}\right) \\
& \text { for some } \left.\alpha_{0}, \alpha_{1} \geq 0, \alpha_{0}+\alpha_{1} \leq 1\right\}, \text { and } \\
\mathcal{R}_{4}^{\prime}=\left\{\left(R_{1}, R_{2}\right):\right. & R_{1} \leq p_{1} \bar{\alpha}_{1} H\left(\alpha_{0} / \bar{\alpha}_{1}\right) \\
& R_{2} \leq H\left(\alpha_{1}\right)-p_{2} \bar{\alpha}_{0} H\left(\alpha_{1} / \bar{\alpha}_{0}\right) \\
& \text { for some } \left.\alpha_{0}, \alpha_{1} \geq 0, \alpha_{0}+\alpha_{1} \leq 1\right\} .
\end{aligned}
$$

To show this, we evaluate $\mathcal{R}_{3}^{\prime}$ and $\mathcal{R}_{4}^{\prime}$ in (3) and note that the rate pairs $\left(C_{1}, 0\right)=(1,0) \in \mathcal{R}_{3}^{\prime}$ and $\left(0, C_{2}\right)=(0,1) \in \mathcal{R}_{4}^{\prime}$. Hence, $\mathcal{C}$ is the convex hull of the union of $\mathcal{R}_{3}^{\prime}$ and $\mathcal{R}_{4}^{\prime}$. The capacity region with state for $\left(p_{1}, p_{2}\right)=(0.5,0.5),(0.7,0.3)$, and $(1,0)$ is plotted in Figure 2. For $\left(p_{1}, p_{2}\right)=(0.5,0.5)$, the two channels are statistically identical, hence the capacity region coincides with the time-division region. For $\left(p_{1}, p_{2}\right)=$ $(1,0)$, the channel reduces to the Blackwell channel with no state [17]. For $\left(p_{1}, p_{2}\right)$ in between these two extreme cases, the capacity region is established by our theorem.

Next consider the following example which is motivated by deterministic approximations of wireless channels. 


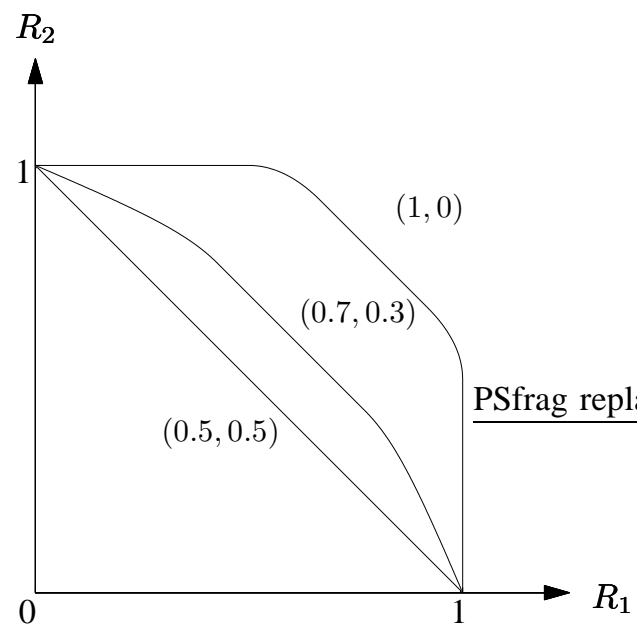

Fig. 2. Capacity region of the Blackwell channel with the state.

Example 2 (Finite-field BC-TDCS). Consider the BC-TDCS with the state known only at the receivers with $\mathbf{X}=$ $\left[\begin{array}{ll}X_{1} & X_{2}\end{array}\right]^{T}$ :

$$
\begin{aligned}
& Y_{1}= \begin{cases}h_{11} X_{1}+h_{12} X_{2} & \text { if } S_{1}=1, \\
h_{21} X_{1}+h_{22} X_{2} & \text { if } S_{1}=2,\end{cases} \\
& Y_{2}= \begin{cases}h_{11} X_{1}+h_{12} X_{2} & \text { if } S_{2}=1, \\
h_{21} X_{1}+h_{22} X_{2} & \text { if } S_{2}=2,\end{cases}
\end{aligned}
$$

where the channel matrix is full-rank, $\mathcal{Y}_{1}=\mathcal{Y}_{2}=\mathcal{X}_{1}=\mathcal{X}_{2}=$ $[0: K-1]$, and the arithmetic is over the finite field.

To compute the capacity region, first note that $C_{1}=\log K$ and $C_{2}=\log K$. Thus,

$$
\begin{aligned}
& \mathcal{R}_{1}=\left\{\left(R_{1}, R_{2}\right): R_{1} \leq \log K, R_{2}=0\right\}, \\
& \mathcal{R}_{2}=\left\{\left(R_{1}, R_{2}\right): R_{1}=0, R_{2} \leq \log K\right\} .
\end{aligned}
$$

To evaluate $\mathcal{R}_{3}$ and $\mathcal{R}_{4}$, we compute $\mathcal{P}_{1}$ and $\mathcal{P}_{2}$. Since

$$
\begin{aligned}
& p_{1} H\left(f_{1}\right)+\bar{p}_{1} I\left(f_{1} ; f_{2}\right)+\lambda \bar{p}_{2} H\left(f_{2} \mid f_{1}\right) \\
& \quad=p_{1} H\left(f_{1}\right)+\bar{p}_{1} H\left(f_{2}\right)+\left(\lambda \bar{p}_{2}-\bar{p}_{1}\right) H\left(f_{2} \mid f_{1}\right) \\
& \quad \leq\left(p_{1}+\lambda \bar{p}_{2}\right) \log K
\end{aligned}
$$

for $\bar{p}_{1} / \bar{p}_{2} \leq \lambda \leq 1$ with equality if $\mathbf{X} \sim \operatorname{Unif}([0$ : $\left.K-1]^{2}\right), \overline{\mathcal{P}}_{1}=\left\{\operatorname{Unif}\left([0: K-1]^{2}\right)\right\}$. Similarly, $\mathcal{P}_{2}=$ $\left\{\operatorname{Unif}\left([0: K-1]^{2}\right)\right\}$. Note that when $\mathbf{X}$ is uniform, $H\left(f_{1}\right)=$ $H\left(f_{2}\right)=H\left(f_{1} \mid f_{2}\right)=H\left(f_{2} \mid f_{1}\right)=\log K$. Hence,

$$
\begin{aligned}
& \mathcal{R}_{3}=\left\{\left(R_{1}, R_{2}\right): R_{1} \leq p_{1} \log K, R_{2} \leq \bar{p}_{2} \log K\right\}, \\
& \mathcal{R}_{4}=\left\{\left(R_{1}, R_{2}\right): R_{1} \leq p_{1} \log K, R_{2} \leq \bar{p}_{2} \log K\right\},
\end{aligned}
$$

and the capacity region is

$$
\mathcal{C}=\operatorname{co}\left\{(0,0),(\log K, 0),(0, \log K),\left(p_{1} \log K, \bar{p}_{2} \log K\right)\right\} .
$$

Figure 3 plots the capacity region for $\left(p_{1}, p_{2}\right)=$ $(0.5,0.5),(0.7,0.4)$, and $(1,0)$. For $\left(p_{1}, p_{2}\right)=(0.5,0.5)$, the two channels are statistically identical and the capacity region coincides with the time-division region. For $\left(p_{1}, p_{2}\right)=(1,0)$, the capacity region is $\left\{\left(R_{1}, R_{2}\right): R_{1} \leq \log K, \quad R_{2} \leq\right.$ $\log K\}$ because the channel matrix is full-rank. For $\left(p_{1}, p_{2}\right)$ in between these two extreme cases, the capacity region is established by our theorem.

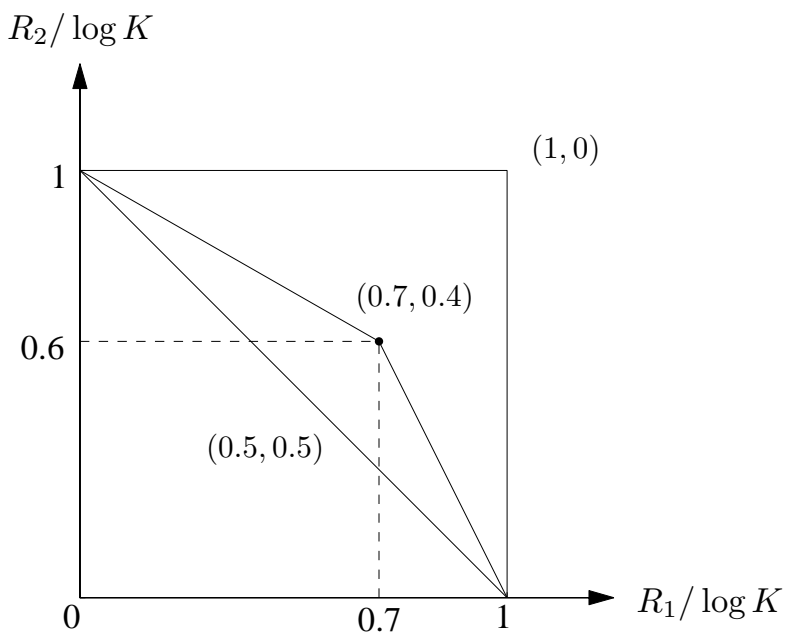

Fig. 3. Capacity region of the Finite Field BC-TDCS.

Connection to wireless channels: Consider the following fading broadcast channel

$$
Y_{j}=\mathbf{H}_{j}^{\dagger} \mathbf{X}+Z_{j} \text { for } j=1,2,
$$

where $\dagger$ denotes the conjugate-transpose, $\mathbf{X}=\left[\begin{array}{ll}X_{1} & X_{2}\end{array}\right]^{T} \in$ $\mathbb{C}^{2 \times 1}, \mathbb{E}\left[\mathbf{X}^{\dagger} \mathbf{X}\right] \leq P, Z_{j} \sim \mathcal{C N}(0,1)$ and the noise sequences $Z_{j i}, j=1,2$ and $i \in[1: n]$, are i.i.d. In addition, for $j=1,2$,

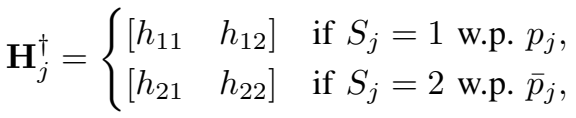

where the channel matrix is in $\mathbb{C}^{2 \times 2}$ and is full rank.

We now show that the degrees of freedom (DoF) of this fading Gaussian broadcast channel, obtained by dividing the maximum sum-rate by $\log P$ and taking the limit, is $p_{1}+\bar{p}_{2}$.

Since the variance of the noise $Z_{j}$ is bounded, the DoF of channel in (5) is equal to that of the BC-TDCS with $Y_{j}=$ $\mathbf{H}_{j}^{\dagger} \mathbf{X}$ for $j=1,2$ [12]. We show that the DoF is achieved when $U_{1}=f_{1}$ and $U_{2}=f_{2}$ are independent and Gaussian with variances $\alpha P$ and $\beta P$ for some $\alpha, \beta>0$ such that

$$
\left[\begin{array}{l}
X_{1} \\
X_{2}
\end{array}\right]=\left[\begin{array}{ll}
h_{11} & h_{12} \\
h_{21} & h_{22}
\end{array}\right]^{-1}\left[\begin{array}{l}
U_{1} \\
U_{2}
\end{array}\right]
$$

satisfy the power constraint. First note that for $\left(R_{1}, R_{2}\right) \in \mathcal{C}$,

$$
\begin{aligned}
& \max \lim _{P \rightarrow \infty} \frac{R_{1}+R_{2}}{\log P} \\
& =\max _{p(\mathbf{X})} \lim _{P \rightarrow \infty} \frac{p_{1} H\left(f_{1}\right)+\bar{p}_{2} H\left(f_{2}\right)+\left(\bar{p}_{1}-\bar{p}_{2}\right) I\left(f_{1} ; f_{2}\right)}{\log P} .
\end{aligned}
$$

Now we show that each term in (6) is maximized with the chosen input. First, $\lim _{P \rightarrow \infty} p_{1} H\left(f_{1}\right) / \log P=$ 
$\lim _{P \rightarrow \infty} p_{1} \log (\alpha P) / \log P=p_{1}$. Now we show that $p_{1}=\max \lim _{P \rightarrow \infty} p_{1} H\left(f_{1}\right) / \log P$. Since $\operatorname{Var}\left(f_{1}\right)=$ $\operatorname{Var}\left(h_{11} X_{1}+h_{12} X_{2}\right)=\left|h_{11}\right|^{2} \gamma P+\left|h_{12}\right|^{2} \bar{\gamma} P+\left(h_{11}^{*} h_{12}+\right.$ $\left.h_{12}^{*} h_{11}\right) \rho \sqrt{\gamma \bar{\gamma}} P$ for some $0 \leq \gamma, \rho \leq 1$ due to the power constraint, $H\left(f_{1}\right) \leq \log \left(\left|h_{11}\right|^{2} \gamma+\left|h_{12}\right|^{2} \bar{\gamma}+\left(h_{11}^{*} h_{12}+\right.\right.$ $\left.\left.h_{12}^{*} h_{11}\right) \rho \sqrt{\gamma \bar{\gamma}}\right)+\log P$. Hence, $\lim _{P \rightarrow \infty} p_{1} H\left(f_{1}\right) / \log P \leq$ $p_{1}$. Similarly, $\lim _{P \rightarrow \infty} \bar{p}_{2} H\left(f_{2}\right) / \log P$ is maximized and is equal to $\bar{p}_{2}$, and $\lim _{P \rightarrow \infty}\left(\bar{p}_{1}-\bar{p}_{2}\right) I\left(f_{1} ; f_{2}\right) / \log P$ is maximized and is equal to 0 . Thus, the following holds:

$$
\begin{aligned}
\max _{p(\mathbf{X})} & \lim _{P \rightarrow \infty} \frac{p_{1} H\left(f_{1}\right)+\bar{p}_{2} H\left(f_{2}\right)+\left(\bar{p}_{1}-\bar{p}_{2}\right) I\left(f_{1} ; f_{2}\right)}{\log P} \\
& =p_{1}+\bar{p}_{2}
\end{aligned}
$$

and the DoF of the fading Gaussian $\mathrm{BC}$ in 5 is $p_{1}+\bar{p}_{2}$.

\section{Proof of the CONVERSE}

The UV bound for the broadcast channel with state known at the receivers states that if a rate pair $\left(R_{1}, R_{2}\right)$ is achievable, then it must lie in the intersection of the regions

$$
\begin{aligned}
\overline{\mathcal{R}}_{1}=\left\{\left(R_{1}, R_{2}\right):\right. & R_{1} \leq I\left(U_{1} ; Y_{1} \mid S\right), \\
& R_{2} \leq I\left(X ; Y_{2} \mid S\right), \\
& R_{1}+R_{2} \leq I\left(U_{1} ; Y_{1} \mid S\right)+I\left(X ; Y_{2} \mid U_{1}, S\right) \\
& \text { for some } \left.p\left(u_{1}, x\right)\right\}, \\
\overline{\mathcal{R}}_{2}=\left\{\left(R_{1}, R_{2}\right):\right. & R_{1} \leq I\left(X ; Y_{1} \mid S\right), \\
& R_{2} \leq I\left(U_{2} ; Y_{2} \mid S\right), \\
& R_{1}+R_{2} \leq I\left(U_{2} ; Y_{2} \mid S\right)+I\left(X ; Y_{1} \mid U_{2}, S\right) \\
& \text { for some } \left.p\left(u_{2}, x\right)\right\} .
\end{aligned}
$$

Denote this outer bound by $\overline{\mathcal{R}}$.

To establish the converse we show that every supporting hyperplane of $\overline{\mathcal{R}}$ intersects $\mathcal{C}$.

Lemma 3. For all $\lambda \geq 0$,

$$
\max _{\left(R_{1}, R_{2}\right) \in \overline{\mathcal{R}}} R_{1}+\lambda R_{2}=\max _{\left(R_{1}, R_{2}\right) \in \mathrm{C}} R_{1}+\lambda R_{2} .
$$

Proof: To prove the lemma for $0 \leq \lambda \leq 1$, consider maximizing $R_{1}+\lambda R_{2}$ over $\left(R_{1}, R_{2}\right) \in \overline{\mathcal{R}}_{1}$.

For any $p\left(u_{1}, x\right), R_{1}+\lambda R_{2}$ such that $\left(R_{1}, R_{2}\right) \in \overline{\mathcal{R}}_{1}$ is maximized when $R_{1}=I\left(U_{1} ; Y_{1} \mid S\right)$ and $R_{2}=I\left(X ; Y_{2} \mid U_{1}, S\right)=$ $H\left(Y_{2} \mid U_{1}, S\right)$. Thus,

$$
\begin{gathered}
\max _{\left(R_{1}, R_{2}\right) \in \overline{\mathcal{R}}_{1}} R_{1}+\lambda R_{2}=\max _{p\left(u_{1}, x\right)} I\left(U_{1} ; Y_{1} \mid S\right)+\lambda H\left(Y_{2} \mid U_{1}, S\right) \\
=\max _{p(x)}\left\{H\left(Y_{1} \mid S\right)+\max _{p\left(u_{1} \mid x\right)}\left\{\lambda H\left(Y_{2} \mid U_{1}, S\right)-H\left(Y_{1} \mid U_{1}, S\right)\right\}\right\} \\
=\max _{p(x)}\left\{p_{1} H\left(f_{1}\right)+\bar{p}_{1} H\left(f_{2}\right)+\max _{p\left(u_{1} \mid x\right)}\left\{\left(\lambda \bar{p}_{2}-\bar{p}_{1}\right) H\left(f_{2} \mid U_{1}\right)\right.\right. \\
\left.\left.+\left(\lambda p_{2}-p_{1}\right) H\left(f_{1} \mid U_{1}\right)\right\}\right\} .
\end{gathered}
$$

For a fixed $p(x)$ only the last two terms in (8) depend on $p\left(u_{1} \mid x\right)$. We now consider different ranges of $0 \leq \lambda \leq 1$.

- If $0 \leq \lambda \leq \bar{p}_{1} / \bar{p}_{2}$, then for any fixed $p(x)$,

$$
\left(\lambda \bar{p}_{2}-\bar{p}_{1}\right) H\left(f_{2} \mid U_{1}\right)+\left(\lambda p_{2}-p_{1}\right) H\left(f_{1} \mid U_{1}\right) \leq 0
$$

with equality if $U_{1}=X$. Thus, (8) can be rewritten as

$$
\begin{aligned}
\max _{\left(R_{1}, R_{2}\right) \in \overline{\mathcal{R}}_{1}} R_{1}+\lambda R_{2} & =\max _{p(x)} p_{1} H\left(f_{1}\right)+\bar{p}_{1} H\left(f_{2}\right) \\
& =\max _{\left(R_{1}, R_{2}\right) \in \mathcal{R}_{1}} R_{1}+\lambda R_{2}
\end{aligned}
$$

- If $\bar{p}_{1} / \bar{p}_{2}<\lambda \leq 1$, then for any fixed $p(x)$,

$$
\begin{aligned}
& \left(\lambda \bar{p}_{2}-\bar{p}_{1}\right) H\left(f_{2} \mid U_{1}\right)+\left(\lambda p_{2}-p_{1}\right) H\left(f_{1} \mid U_{1}\right) \\
& =(\lambda-1) H\left(f_{1} \mid U_{1}\right) \\
& \quad+\left(\lambda \bar{p}_{2}-\bar{p}_{1}\right)\left(H\left(f_{2} \mid f_{1}, U_{1}\right)-H\left(f_{1} \mid f_{2}, U_{1}\right)\right) \\
& \leq\left(\lambda \bar{p}_{2}-\bar{p}_{1}\right) H\left(f_{2} \mid f_{1}\right)
\end{aligned}
$$

with equality if $U_{1}=f_{1}$. Thus, (8) can be rewritten as

$$
\begin{aligned}
& \max _{\left(R_{1}, R_{2}\right) \in \overline{\mathcal{R}}_{1}} R_{1}+\lambda R_{2} \\
& \quad=\max _{p(x)} p_{1} H\left(f_{1}\right)+\bar{p}_{1} I\left(f_{1} ; f_{2}\right)+\lambda \bar{p}_{2} H\left(f_{2} \mid f_{1}\right) \\
& \quad=\max _{\left(R_{1}, R_{2}\right) \in \mathcal{R}_{3}} R_{1}+\lambda R_{2} .
\end{aligned}
$$

Thus, $\max _{\left(R_{1}, R_{2}\right) \in \mathcal{C}} R_{1}+\lambda R_{2} \geq \max _{\left(R_{1}, R_{2}\right) \in \overline{\mathcal{R}}} R_{1}+\lambda R_{2}$ for $0 \leq \lambda \leq 1$. Equality in the lemma holds because $\mathcal{C} \subseteq \overline{\mathcal{R}}$. The proof for $\lambda>1$ follows similarly (see Appendix B).

The proof of the converse is completed using Lemma 2.

\section{CONCLUSION}

We established the capacity region of the BC-TDCS channel when the state is known only at the receivers. This channel does not belong to any class of broadcast channels for which the capacity was previously known. There are several open problems that would be interesting to explore further, including: What is the capacity region of the BC-TDCS with common message when the state is known only at the receivers? What is the capacity region when each channel component is selected from a set of more than two deterministic channel states?

\section{ACKNOWLEDGMEnTS}

The authors thank Chandra Nair and Young-Han Kim for comments that have improved the readability of this paper.

\section{REFERENCES}

[1] H. Kim, Y.-K. Chia, and A. El Gamal, "A note on broadcast channels with stale state information at the transmitter," CoRR, vol. abs/1309.7437, 2013.

[2] A. El Gamal and Y. H. Kim, Network Information Theory, 1st ed. Cambridge University Press, 2011.

[3] Y. Geng, A. Gohari, C. Nair, and Y. Yu, "On marton's inner bound and its optimality for classes of product broadcast channels," Information Theory, IEEE Transactions on, vol. 60, no. 1, pp. 22-41, 2014.

[4] A. Jafarian and S. Vishwanath, "The two-user gaussian fading broadcast channel," in Information Theory Proceedings (ISIT), 2011 IEEE International Symposium on, 2011, pp. 2964-2968.

[5] Y. Liang and A. Goldsmith, "Rate regions and optimal power allocation for td fading broadcast channels without csit," in Allerton, Monticello IL, 2005.

[6] D. Tse and R. Yates, "Fading broadcast channels with state information at the receivers," Information Theory, IEEE Transactions on, vol. 58, no. 6, pp. 3453-3471, 2012.

[7] E. Abbe and L. Zheng, "Coding along hermite polynomials for gaussian noise channels," in Information Theory, 2009. ISIT 2009. IEEE International Symposium on, 2009, pp. 1644-1648. 
[8] R. Khosravi-Farsani and F. Marvasti, "Capacity bounds for multiuser channels with non-causal channel state information at the transmitters,' in Information Theory Workshop (ITW), 2011 IEEE, 2011, pp. 195-199.

[9] A. Lapidoth and L. Wang, "The state-dependent semideterministic broadcast channel," Information Theory, IEEE Transactions on, vol. 59, no. 4, pp. 2242-2251, 2013.

[10] P. Larsson and N. Johansson, "Multi-user arq," in Vehicular Technology Conference, 2006. VTC 2006-Spring. IEEE 63rd, vol. 4, 2006, pp. 20522057.

[11] L. Georgiadis and L. Tassiulas, "Broadcast erasure channel with feedback - capacity and algorithms," in Network Coding, Theory, and Applications, 2009. NetCod '09. Workshop on, 2009, pp. 54-61.

[12] M. Maddah-Ali and D. Tse, "Completely stale transmitter channel state information is still very useful," in Information Theory, IEEE Transactions on, vol. 58, no. 7, 2012, pp. 4418-4431.

[13] O. Shayevitz and M. Wigger, "On the capacity of the discrete memoryless broadcast channel with feedback," IEEE Trans. Inf. Theory, vol. 59, no. 3, pp. 1329-1345, 2013.

[14] K. Marton, "A coding theorem for the discrete memoryless broadcast channel," Information Theory, IEEE Transactions on, vol. 25, no. 3, pp. 306-311, 1979.

[15] C. Nair and A. El Gamal, "An outer bound to the capacity region of the broadcast channel," in Information Theory, 2006 IEEE International Symposium on, 2006, pp. 2205-2209.

[16] H. G. Egglestone, Convexity. Cambridge University Press, Cambridge, 1958.

[17] D. Blackwell, L. Breiman, and A. J. Thomasian, "Proof of shannon's transmission theorem for finite-state indecomposable channels," The Annals of Mathematical Statistics, vol. 29, no. 4, pp. pp. 1209-1220, Dec. 1958.

\section{APPENDIX A}

\section{PROOF OF LEMMA 1}

We prove the lemma for $0 \leq \lambda \leq 1$. The proof for $\lambda>1$ follows similarly. First we show that for $0 \leq \lambda \leq 1$,

$$
\begin{aligned}
& \max _{\left(R_{1}, R_{2}\right) \in \mathcal{C}} R_{1}+\lambda R_{2}=\max _{i=1,2,3,4}\left\{\max _{\left(R_{1}, R_{2}\right) \in \mathcal{R}_{i}^{\prime}} R_{1}+\lambda R_{2}\right\} \\
& \stackrel{(a)}{=} \max _{i=1,3}\left\{\max _{\left(R_{1}, R_{2}\right) \in \mathcal{R}_{i}^{\prime}} R_{1}+\lambda R_{2}\right\} \\
& \stackrel{(b)}{=}\left\{\begin{array}{l}
\max _{\left(R_{1}, R_{2}\right) \in \mathcal{R}_{1}} R_{1}+\lambda R_{2} \text { if } 0 \leq \lambda \leq \bar{p}_{1} / \bar{p}_{2}, \\
\max _{\left(R_{1}, R_{2}\right) \in \mathcal{R}_{3}} R_{1}+\lambda R_{2} \text { if } \bar{p}_{1} / \bar{p}_{2}<\lambda \leq 1 .
\end{array}\right.
\end{aligned}
$$

The equality in $(a)$ holds because

$$
\begin{aligned}
& \quad \max _{\left(R_{1}, R_{2}\right) \in \mathcal{R}_{3}^{\prime}} R_{1}+\lambda R_{2} \\
& \quad=\max _{p(x)} I\left(f_{1} ; Y_{1} \mid S\right)+\lambda I\left(f_{2} ; Y_{2} \mid S\right)-\lambda I\left(f_{1} ; f_{2}\right) \\
& \quad \geq \max _{p(x)} I\left(f_{1} ; Y_{1} \mid S\right)-I\left(f_{1} ; f_{2}\right)+\lambda I\left(f_{2} ; Y_{2} \mid S\right) \\
& \quad=\max _{\left(R_{1}, R_{2}\right) \in \mathcal{R}_{4}^{\prime}} R_{1}+\lambda R_{2}, \text { and } \\
& \quad \max _{\left(R_{1}, R_{2}\right) \in \mathcal{R}_{4}^{\prime}} R_{1}+\lambda R_{2} \\
& \quad=\max _{p(x)} \lambda p_{2} H\left(f_{1}\right)+\lambda \bar{p}_{2} H\left(f_{2}\right)+\left(p_{1}-\lambda p_{2}\right) H\left(f_{1} \mid f_{2}\right) \\
& \quad \geq \max _{p(x)} \lambda p_{2} H\left(f_{1}\right)+\lambda \bar{p}_{2} H\left(f_{2}\right)=\underset{\left(R_{1}, R_{2}\right) \in \mathcal{R}_{2}^{\prime}}{\max _{1}+\lambda R_{2} .}
\end{aligned}
$$

To derive the equality in $(b)$ note that

$$
\begin{aligned}
& \quad \max _{\left(R_{1}, R_{2}\right) \in \mathcal{R}_{1}^{\prime}} R_{1}+\lambda R_{2}=\max _{p(x)}\left\{p_{1} H\left(f_{1}\right)+\bar{p}_{1} H\left(f_{2}\right)\right\}, \\
& \max _{\left(R_{1}, R_{2}\right) \in \mathcal{R}_{3}^{\prime}} R_{1}+\lambda R_{2} \\
& \quad=\max _{p(x)}\left\{p_{1} H\left(f_{1}\right)+\bar{p}_{1} H\left(f_{2}\right)+\left(\lambda \bar{p}_{2}-\bar{p}_{1}\right) H\left(f_{2} \mid f_{1}\right)\right\} .
\end{aligned}
$$

For $0 \leq \lambda \leq \bar{p}_{1} / \bar{p}_{2}, \max _{\left(R_{1}, R_{2}\right) \in \mathcal{R}_{1}^{\prime}} R_{1}+\lambda R_{2} \geq$

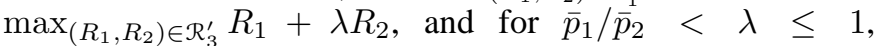

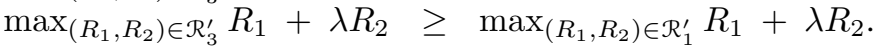

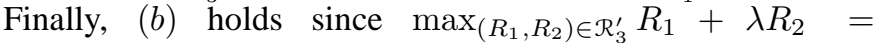
$\max _{\left(R_{1}, R_{2}\right) \in \mathcal{R}_{3}} R_{1}+\lambda R_{2}$ for $\bar{p}_{1} / \bar{p}_{2}<\lambda \leq 1$ and $\mathcal{R}_{1}^{\prime}=\mathcal{R}_{1}$.

Thus, for $0 \leq \lambda \leq 1, \max _{\left(R_{1}, R_{2}\right) \in \mathcal{C}_{0}} R_{1}+\lambda R_{2} \geq$ $\max _{\left(R_{1}, R_{2}\right) \in \mathcal{C}} R_{1}+\lambda R_{2}$. Finally, equality holds because $\mathcal{C}_{0} \subseteq$ e.

\section{APPENDIX B}

PROOF OF LEMMA 3 FOR $\lambda>1$

For $\lambda>1$, we consider the equivalent maximization problem: $\max _{\left(R_{1}, R_{2}\right) \in \overline{\mathcal{R}}_{2}} \lambda^{-1} R_{1}+R_{2}$ for $\lambda^{-1}<1$.

For any $p\left(u_{2}, x\right)$, among the $\left(R_{1}, R_{2}\right) \in \overline{\mathcal{R}}_{2}, \lambda^{-1} R_{1}+R_{2}$ is maximized when $R_{2}=I\left(U_{2} ; Y_{2} \mid S\right)$ and $R_{1}=H\left(Y_{1} \mid U_{2}, S\right)$. Thus,

$$
\begin{aligned}
& \max _{\left(R_{1}, R_{2}\right) \in \overline{\mathcal{R}}_{2}} \lambda^{-1} R_{1}+R_{2} \\
& =\max _{p\left(u_{2}, x\right)} \lambda^{-1} H\left(Y_{1} \mid U_{2}, S\right)+I\left(U_{2} ; Y_{2} \mid S\right) \\
& =\max _{p(x)}\left\{H\left(Y_{2} \mid S\right)+\max _{p\left(u_{2} \mid x\right)}\left\{\lambda^{-1} H\left(Y_{1} \mid U_{2}, S\right)\right.\right. \\
& \left.\left.\quad \quad-H\left(Y_{2} \mid U_{2}, S\right)\right\}\right\} \\
& =\max _{p(x)}\left\{p_{2} H\left(f_{1}\right)+\bar{p}_{2} H\left(f_{2}\right)\right. \\
& \quad+\max _{p\left(u_{2} \mid x\right)}\left\{\left(\lambda^{-1} \bar{p}_{1}-\bar{p}_{2}\right) H\left(f_{2} \mid U_{2}\right)\right. \\
& \left.\left.\quad+\left(\lambda^{-1} p_{1}-p_{2}\right) H\left(f_{1} \mid U_{2}\right)\right\}\right\} .
\end{aligned}
$$

For a fixed $p(x)$, only the last two terms in (9) depend on $p\left(u_{2} \mid x\right)$. We now consider different ranges of $\lambda>1$.

- If $\lambda>p_{1} / p_{2}$, then for any fixed $p(x)$,

$$
\left(\lambda^{-1} \bar{p}_{1}-\bar{p}_{2}\right) H\left(f_{2} \mid U_{2}\right)+\left(\lambda^{-1} p_{1}-p_{2}\right) H\left(f_{1} \mid U_{2}\right) \leq 0
$$

with equality if $U_{2}=X$. Then, (9) can be expressed as

$$
\begin{aligned}
\max _{\left(R_{1}, R_{2}\right) \in \overline{\mathcal{R}}_{2}} R_{1}+\lambda R_{2} & =\max _{p(x)} \lambda p_{2} H\left(f_{1}\right)+\lambda \bar{p}_{2} H\left(f_{2}\right) \\
& =\max _{\left(R_{1}, R_{2}\right) \in \mathcal{R}_{2}} R_{1}+\lambda R_{2} .
\end{aligned}
$$

- If $1<\lambda \leq p_{1} / p_{2}$, then for any fixed $p(x)$,

$$
\begin{aligned}
& \quad\left(\lambda^{-1} \bar{p}_{1}-\bar{p}_{2}\right) H\left(f_{2} \mid U_{2}\right)+\left(\lambda^{-1} p_{1}-p_{2}\right) H\left(f_{1} \mid U_{2}\right) \\
& =\left(\lambda^{-1}-1\right) H\left(f_{2} \mid U_{2}\right) \\
& \quad \quad+\left(\lambda^{-1} p_{1}-p_{2}\right)\left\{H\left(f_{1} \mid f_{2}, U_{2}\right)-H\left(f_{2} \mid f_{1}, U_{2}\right)\right\} \\
& \leq\left(\lambda^{-1} p_{1}-p_{2}\right) H\left(f_{1} \mid f_{2}\right)
\end{aligned}
$$

with equality if $U_{2}=f_{2}$. Then, (9) can be expressed as

$$
\begin{aligned}
& \max _{\left(R_{1}, R_{2}\right) \in \overline{\mathcal{R}}_{2}} R_{1}+\lambda R_{2} \\
& \quad=\max _{p(x)} p_{1} H\left(f_{1} \mid f_{2}\right)+\lambda p_{2} I\left(f_{1} ; f_{2}\right)+\lambda \bar{p}_{2} H\left(f_{2}\right) \\
& \quad=\max _{\left(R_{1}, R_{2}\right) \in \mathcal{R}_{4}} R_{1}+\lambda R_{2} .
\end{aligned}
$$

Thus, $\max _{\left(R_{1}, R_{2}\right) \in \mathcal{C}} R_{1}+\lambda R_{2} \geq \max _{\left(R_{1}, R_{2}\right) \in \overline{\mathcal{R}}} R_{1}+\lambda R_{2}$ for $\lambda>1$. Finally, equality holds because $\mathcal{C} \subseteq \mathcal{R}$. 